\title{
FUNCTIONS OF EXPONENTIAL TYPE
}

\author{
BY \\ Q. I. RAHMAN
}

1. Introduction. Let $f(z)$ be an entire function of exponential type $\tau$ (i.e. a function of order 1 and type $\leqq \tau$, or a function of order less than 1$)$, with $|f(x)| \leqq M$ on the real axis. It is well known (for references see [2, pp. 82, 206]) that then

$$
\begin{aligned}
|f(x+i y)| & \leqq M e^{\tau|y|}, \\
\left|f^{\prime}(x)\right| & \leqq M \tau .
\end{aligned}
$$

Duffin and Schaeffer [8] refined the first of these inequalities for functions that are real on the real axis by showing that in this case

$$
|f(x+i y)| \leqq M \cosh \tau y .
$$

On the other hand, Boas [3] proved that if $f(z) \neq 0$ for $y>0$ and $h_{f}(\pi / 2)=0\left(h_{f}(\theta)=\right.$ $\lim \sup _{r \rightarrow \infty}\left(\log \left|f\left(r e^{i \theta}\right)\right| / r\right)$ denotes the indicator of $\left.f(z)\right)$, then for $y<0$

$$
|f(x+i y)| \leqq(M / 2)\left(e^{\tau|y|}+1\right),
$$

and for all real $x$

$$
\left|f^{\prime}(x)\right| \leqq M \tau / 2 .
$$

In this paper we develop a unified method for arriving at these inequalities. In spite of being extremely simple, the method turns out to be very useful and effective. Not only does it give simpler proofs of the above results but yields interesting generalizations as well. We use the same idea to prove some other inequalities later in the paper.

2. The following result is well known (for references see [2, p. 82]).

LEMMA 1. If $f(z)$ is regular and of exponential type in the upper half plane, $h_{f}(\pi / 2) \leqq c$, and $|f(x)| \leqq M,-\infty<x<\infty$, then $|f(x+i y)| \leqq M e^{c y}, \quad-\infty<x<\infty$, $0 \leqq y<\infty$.

Lemma 2. Let $\phi(z)$ be an entire function of exponential type $\tau$ which does not vanish in the upper half plane and $h_{\phi}(\pi / 2)=0$. If $\psi(z)=e^{i z z} \phi(z)$, then for $\operatorname{Im} z<0$

$$
|\phi(z)| \leqq|\psi(z)|
$$

and for all real $x$

$$
\left|\phi^{\prime}(x)\right| \leqq\left|\psi^{\prime}(x)\right| .
$$

Received by the editors August 7, 1967. 
Proof of Lemma 2. The function $\Phi(z)=\phi(z) e^{-i \tau z / 2}$ has no zeros for $y>0$ and $h_{\Phi}(+\pi / 2)=\tau / 2 \geqq h_{\Phi}(-\pi / 2)$. By a theorem of Levin [2, p. 129] we have $|\Phi(z)|$ $\leqq|\Phi(\bar{z})|$ for $y<0$. Thus for $\operatorname{Im} z<0$

This is (2.1).

$$
\begin{aligned}
|\phi(z)| & \leqq\left|e^{i \tau z / 2}\right|\left|\phi(\bar{z}) e^{-i \tau \bar{z} / 2}\right|=\left|e^{i \tau z / 2}\right|\left|\phi(z) e^{i \tau z / 2}\right| \\
& =\left|\phi(z) e^{i \tau z}\right|=|\psi(z)| .
\end{aligned}
$$

For the proof of (2.2), we may assume that $\tau>0$. In fact, if $\tau=0$ then $\psi(z)$ reduces to $\phi(z)$ and there is equality in (2.2).

Note that the function $\psi(z)$ has no zeros for $\operatorname{Im} z<0$. Besides, $h_{\psi}(-\pi / 2)=\tau$ and $h_{\psi}(\pi / 2) \leqq 0$, i.e. $h_{\psi}(-\pi / 2)>h_{\psi}(\pi / 2)$. Hence $\psi(z)$ is of order 1 , type $\sigma \geqq \tau$, and belongs to the class $P$ discussed in [2, p. 129]. Since $|\phi(x)|=|\psi(x)|$ for $-\infty<x<\infty$, it follows by another theorem of Levin [2, p. 226] that for all real $x,\left|\phi^{\prime}(x)\right| \leqq\left|\psi^{\prime}(x)\right|$.

3.1. Proof of (1.3). If $f(z)$ is a constant there is nothing to prove. So let us assume that $f(z)$ is not a constant. Let

$$
\limsup _{r \rightarrow \infty} \frac{\log \left|f\left(r e^{i \pi / 2}\right)\right|}{r}=c \leqq \tau .
$$

Since $f(z)$ is assumed to be bounded on the real axis and is real for real $z, c$ must be positive. Otherwise $f(z)$ will be bounded in the whole plane by Lemma 1 and must therefore be a constant.

If $|\lambda|>1$ then the function $F(z)=e^{i c z} f(z) /(\lambda M)$ is an entire function of exponential type, $h_{F}(\pi / 2)=0$ and $|F(x)|<1,-\infty<x<\infty$. By Lemma 1 , it follows that $|F(z)|<1$ for $\operatorname{Im} z \geqq 0$. Hence the function $F_{1}(z)=f(z)-\lambda M e^{-i c z} \neq 0$ for $\operatorname{Im} z>0$. The function $e^{i c z} F_{1}(z)$ is of exponential type $2 c$ and satisfies the conditions of Lemma 2. Therefore, for $\operatorname{Im} z<0$ we have

or

$$
\left|e^{i c z} F_{1}(z)\right| \leqq\left|e^{2 i c z} e^{-i c z} \bar{F}_{1}(z)\right|
$$

$$
\left|F_{1}(z)\right| \leqq\left|F_{1}(\bar{z})\right| \text {. }
$$

If $\arg \lambda$ is so chosen that $\left|f(\bar{z})-\lambda M e^{-i c \bar{z}}\right|=\left|\lambda M e^{-i c \bar{z}}\right|-|f(\bar{z})|$ then for $\operatorname{Im} z<0$

$$
|f(z)|-|\lambda| M e^{c y} \leqq\left|f(z)-\lambda M e^{-i c z}\right| \leqq\left|f(\bar{z})-\lambda M e^{-i c \bar{z}}\right|=|\lambda| M e^{-c y}-|f(\bar{z})| .
$$

Since $f(z)$ is real for real $z$, we have $|f(z)|=|f(\bar{z})|$ and this gives

$$
|f(z)| \leqq M|\lambda| \cosh c y \leqq M|\lambda| \cosh \tau y
$$

for all $z$. Making $|\lambda| \rightarrow 1$ we get the result.

3.2. Proof of (1.4) and (1.5). Let $\omega(z)=e^{i \tau z} \bar{f}(z)$. By Lemma 2 it follows that for $\operatorname{Im} z<0$

$$
|f(z)| \leqq|\omega(z)|
$$

and for all real $x$

$$
\left|f^{\prime}(x)\right| \leqq\left|\omega^{\prime}(x)\right|
$$


Since $h_{f}(\pi / 2)=0$ and $|f(x)| \leqq M$ we have $|f(z)| \leqq M$ for $\operatorname{Im} z>0$. Hence for $|\lambda|>1$ the function $F_{2}(z)=f(z)-\lambda M$ is an entire function of exponential type $\tau$ such that $h_{F_{2}}(\pi / 2)=0, F_{2}(z) \neq 0$ for $\operatorname{Im} z>0$. Applying (2.1) and (2.2) to the functions $F_{2}(z)$ and $\Omega(z)=e^{i z z} \bar{F}_{2}(z)$ we get

$$
\left|F_{2}(z)\right| \leqq|\Omega(z)|
$$

for $\operatorname{Im} z<0$ and

$$
\left|F_{2}^{\prime}(x)\right| \leqq\left|\Omega^{\prime}(x)\right|
$$

for $-\infty<x<\infty$. But

$$
\Omega(z)=e^{i \tau z} \bar{f}(z)-M \bar{\lambda} e^{i \tau z}=\omega(z)-M \bar{\lambda} e^{i \tau z} ;
$$

therefore

$$
|f(z)-\lambda M| \leqq\left|\omega(z)-M \bar{\lambda} e^{i \tau z}\right|
$$

for $\operatorname{Im} z<0$ and

$$
\left|f^{\prime}(x)\right| \leqq\left|\omega^{\prime}(x)-M \lambda i \tau e^{i \tau x}\right|
$$

for $-\infty<x<\infty$. With a suitable choice of $\arg \lambda$ in each case we shall obtain

$$
|f(z)-\lambda M| \leqq M|\lambda| e^{\tau|y|}-|\omega(z)|
$$

for $y=\operatorname{Im} z<0$ and

$$
\left|f^{\prime}(x)\right| \leqq M|\lambda| \tau-\left|\omega^{\prime}(x)\right|
$$

for $-\infty<x<\infty$. Hence, for $\operatorname{Im} z<0$

$$
|f(z)|+|\omega(z)| \leqq M|\lambda| e^{\tau|y|}+M|\lambda|
$$

and for $-\infty<x<\infty$

$$
\left|f^{\prime}(x)\right|+\left|\omega^{\prime}(x)\right| \leqq M|\lambda| \tau .
$$

Finally, making $|\lambda| \rightarrow 1$ we get

$$
|f(z)|+|\omega(z)| \leqq M\left(e^{\tau|y|}+1\right)
$$

for $y<0$ and

$$
\left|f^{\prime}(x)\right|+\left|\omega^{\prime}(x)\right| \leqq M \tau
$$

for $-\infty<x<\infty$. Note that (3.11) together with (3.1) implies (1.4), whereas (1.5) follows from (3.2) and (3.12).

REMARK 1. Let $f(z)$ be an entire function of order 1 type $\tau$ such that $h_{f}(\pi / 2) \leqq 0$ and $f(z) \neq 0$ for $\operatorname{Im} z<0$. Suppose further that $\sup _{-\infty<x<\infty}|f(x)|=M$ on the real axis. Since then $h_{f}(-\pi / 2)=\tau$ it follows that $\omega(z)=e^{i \tau z} \bar{f}(z)$ is an entire function of order 1 type $\tau$ and $h_{\omega}(\pi / 2)=0$. Besides, $\omega(z) \neq 0$ for $\operatorname{Im} z>0$. Hence from (1.5) we have

$$
\left|i \tau e^{i \tau x} \bar{f}(x)+e^{i \tau x} \bar{f}^{\prime}(x)\right| \leqq M \tau / 2,
$$


for all real $x$. Now let $\varepsilon>0$ be fixed. We can find a real number $x_{0}$ such that $\left|f\left(x_{0}\right)\right|>M-\varepsilon$. From (3.13) we get

$$
\tau(M-\varepsilon)-\left|f^{\prime}\left(x_{0}\right)\right| \leqq M \tau / 2
$$

and therefore

$$
\sup _{-\infty<x<\infty}\left|f^{\prime}(x)\right| \geqq\left|f^{\prime}\left(x_{0}\right)\right| \geqq M \tau / 2-\varepsilon \tau .
$$

Since $\varepsilon>0$ is arbitrary we in fact have $\sup _{-\infty<x<\infty}\left|f^{\prime}(x)\right| \geqq M \tau / 2$.

Thus we can state the following:

THEOREM 1. If $f(z)$ is an entire function of exponential type $\tau$ such that

$$
\sup _{-\infty<x<\infty}|f(x)|=M, \quad h_{f}(-\pi / 2)=\tau, \quad h_{f}(\pi / 2) \leqq 0, \quad \text { and } \quad f(z) \neq 0
$$

for $y<0$, then $\sup _{-\infty<x<\infty}\left|f^{\prime}(x)\right| \geqq M \tau / 2$.

Combining this result with (1.5) we obtain:

COROllary 1. If $f(z)$ is an entire function of exponential type $\tau$ with

$$
\sup _{-\infty<x<\infty}|f(x)|=M, \quad h_{f}(-\pi / 2)=\tau, \quad h_{f}(\pi / 2)=0,
$$

and $f(z)$ has all its zeros on the real axis, then $\sup _{-\infty<x<\infty}\left|f^{\prime}(x)\right|=M \tau / 2$.

3.3. Let $L^{p}, 1 \leqq p<\infty$ denote the class of measurable functions $f$, for which $\int_{-\infty}^{\infty}|f(x)|^{p} d x$ is finite. Now, if $f(z)$ is an entire function of exponential type $\tau$ belonging to $L^{p}, 1 \leqq p<\infty$ on the real axis then [2, p. 211],

$$
\left\|f^{\prime}\right\|_{p}=\left(\int_{-\infty}^{\infty}\left|f^{\prime}(x)\right|^{p} d x\right)^{1 / p} \leqq \tau\left(\int_{-\infty}^{\infty}|f(x)|^{p} d x\right)^{1 / p}=\tau\|f\|_{p}
$$

E. M. Stein has shown that this inequality is also implied by (1.2). In fact, he proves the following general [14, Theorem 1].

THEOREM A. Let $T$ and $S$ be linear operators, each satisfying the following conditions:

(1) $T$ is defined on $B_{\imath}$ (the set of entire functions of exponential type $\tau$ bounded on the real line), and commutes with translations.

(2) There exists a constant $A_{\tau}$ so that $\|T(f)\|_{\infty} \leqq A_{\tau}\|f\|_{\infty}$ where

$$
\|\vartheta\|_{\infty}=\sup _{-\infty<x<\infty}|\vartheta(x)| \text {. }
$$

Now suppose that for some constant $\lambda_{\tau}$,

(a) $\|T(f)\|_{\infty} \leqq \lambda_{\tau}\|S(f)\|_{\infty}$, if $f \in B_{\tau}$; then $\|T(f)\|_{p} \leqq \lambda_{\tau}\|S(f)\|_{p}$, if $f \in E_{\tau} \cap L^{p}$ $1 \leqq p<\infty, E_{\tau}$ being the set of entire functions of exponential type $\tau$.

(b) Moreover, if $\left|T f\left(x_{0}\right)\right|=\lambda_{\tau}\|S(f)\|_{\infty}$, for some $x_{0}$ implies that $f(z)=a e^{i \tau z}+b e^{-i \tau z}$, then $\|T(f)\|_{p}=\lambda_{\tau}\|S(f)\|_{p}$ implies $f=0$, if $f \in E_{\tau} \cap L^{p}, 1 \leqq p<\infty$. 
Thus $T=d / d x$ and $S=I$ (where $I=$ identity operator and $\lambda_{\tau}=\tau$ ) give (3.14) as a consequence of (1.2). It would be interesting to obtain the extension of (1.5) to $L^{p}, 1 \leqq p<\infty$, with the help of Theorem A.

THEOREM 2. If $f(z)$ is an entire function of exponential type $\tau$ belonging to $L^{p}$, $1 \leqq p<\infty$ on the real axis, $h_{f}(\pi / 2)=0$ and $f(z) \neq 0$ for $y>0$, then for $p \geqq 1$ we have

$$
\left\|f^{\prime}\right\|_{p} \leqq C_{p}^{1 / p} \tau\|f\|_{p}
$$

where

$$
C_{p}=2 \pi / \int_{0}^{2 \pi}\left|1+e^{i \alpha}\right|^{p} d \alpha=2^{-p} \sqrt{ } \pi \Gamma\left(\frac{1}{2} p+1\right) / \Gamma\left(\frac{1}{2} p+\frac{1}{2}\right) .
$$

For another proof of this result see [12].

Proof of Theorem 2. Let $f(z)$ be an entire function of exponential type $\tau$ bounded by $M$ on the real axis. If $f^{*}(z)=e^{i \tau z} f(z)$ then $h_{f^{*}}(\pi / 2) \leqq 0$. Since $\left|f^{*}(x)\right| \leqq M$ for $-\infty<x<\infty$ it follows from Lemma 1 that $\left|f^{*}(z)\right| \leqq M$ in the upper half plane. Hence for every $\lambda$ such that $|\lambda|>1$ the function $f_{+}(z)=f^{*}(z)-\lambda M$ does not vanish in the upper half plane. We claim that if

$$
g(z) \stackrel{\perp}{=} e^{2 i \tau z} \bar{f}^{*}(z)-\bar{\lambda} M e^{2 i \tau z}=e^{i \tau z} \bar{f}(z)-\lambda M e^{2 i \tau z}=\omega(z)-\lambda M e^{2 i \tau z}
$$

then $\left|f_{+}^{\prime}(x)\right| \leqq\left|g^{\prime}(x)\right|$ for $-\infty<x<\infty$. This is obvious if $\tau=0$ for then $g(x)=\bar{f}_{+}(x)$ $-\bar{\lambda} M$. So let $\tau>0$. It is clear that $g(z)$ does not have zeros in the lower half plane. Besides, it is of order 1 and type $2 \tau$. That it is of exponential type $2 \tau$ is obvious. But for $y<0$

$$
|g(i y)| \geqq|\lambda| M e^{2 \tau|y|}-e^{\tau|y|}|f(-i y)| \geqq(|\lambda|-1) M e^{2 \tau|y|}
$$

by (1.1). Hence $h_{g}(-\pi / 2) \geqq 2 \tau$ and $g(z)$ is, in fact, of order 1 type $2 \tau$. Now since $\left|f_{+}(x)\right|=|g(x)|$ for $-\infty<x<\infty$ it follows from the theorem of Levin [2, p. 226] which we used for the proof of (2.2) that

$$
\left|f_{+}^{\prime}(x)\right| \leqq\left|g^{\prime}(x)\right|, \quad-\infty<x<\infty
$$

But

$$
\begin{aligned}
\left|f_{+}^{\prime}(x)\right| & =\left|i \tau f(x)+f^{\prime}(x)\right| \\
\left|g^{\prime}(x)\right| & =\left|i \tau \bar{f}(x)+\bar{f}^{\prime}(x)-2 \lambda M i \tau e^{i \tau x}\right|=\left|-i \tau f(x)+f^{\prime}(x)+2 i \lambda M e^{-i \tau x}\right| .
\end{aligned}
$$

Hence

$$
\left|i \tau f(x)+f^{\prime}(x)\right| \leqq\left|-i \tau f(x)+f^{\prime}(x)+2 i \lambda M \tau e^{-i \tau x}\right|, \quad-\infty<x<\infty .
$$

Choosing arg $\lambda$ such that

$$
\left|-i \tau f(x)+f^{\prime}(x)+2 i \lambda M e^{-i \tau x}\right|=2|\lambda| M \tau-\left|-i \tau f(x)+f^{\prime}(x)\right|
$$

we get

$$
\left|i \tau f(x)+f^{\prime}(x)\right|+\left|-i \tau f(x)+f^{\prime}(x)\right| \leqq 2 M \tau, \quad-\infty<x<\infty .
$$


Thus, for every $\alpha$ in $[0,2 \pi)$, we have

$$
\left|i \tau f(x)+f^{\prime}(x)+e^{i \alpha}\left\{-i \tau f(x)+f^{\prime}(x)\right\}\right| \leqq 2 M \tau, \quad-\infty<x<\infty .
$$

If $D \equiv d / d x$ then the above inequality can be written as

$$
\left|\left\{\left(e^{i \alpha}+1\right) D+\left(1-e^{i \alpha}\right) i \tau\right\} f(x)\right| \leqq 2 M \tau, \quad-\infty<x<\infty .
$$

Clearly, $\left(e^{i \alpha}+1\right) D+\left(1-e^{i \alpha}\right) i \tau$ is a linear operator $T$ satisfying the conditions of Theorem $A$ and we can write (3.17) as

$$
\|T(f)\|_{\infty} \leqq 2 \tau\|I(f)\|_{\infty}
$$

where $I$ is the identity operator. Hence from Theorem A we have

$$
\int_{-\infty}^{\infty}\left|i \tau f(x)+f^{\prime}(x)+e^{i \alpha}\left\{-i \tau f(x)+f^{\prime}(x)\right\}\right|^{p} d x \leqq(2 \tau)^{p} \int_{-\infty}^{\infty}|f(x)|^{p} d x
$$

for $f \in E_{\tau} \cap L_{p}, 1 \leqq p<\infty$. If, in particular, $h_{f}(\pi / 2)=0$ then $f_{1}(z)=e^{-i \tau z / 2} f(z)$ is of exponential type $\tau / 2$ and on applying (3.18) to $f_{1}(z)$ we get

or

$$
\begin{aligned}
\int_{-\infty}^{\infty} \mid i \frac{\tau}{2} f_{1}(x)+f_{1}^{\prime}(x)+e^{i \alpha}\left\{-i \frac{\tau}{2} f_{1}(x)\right. & \left.+f_{1}^{\prime}(x)\right\}\left.\right|^{p} d x \\
& \leqq \tau^{p} \int_{-\infty}^{\infty}\left|f_{1}(x)\right|^{p} d x, \quad(p \geqq 1)
\end{aligned}
$$

$$
\int_{-\infty}^{\infty}\left|e^{-i \tau x / 2} f^{\prime}(x)+e^{i \alpha}\left\{-i \tau e^{-i \tau x / 2} f(x)+e^{-i \tau x / 2} f^{\prime}(x)\right\}\right|^{p} d x \leqq \tau^{p} \int_{-\infty}^{\infty}|f(x)|^{p} d x
$$

for $p \geqq 1$. Consequently

$$
\int_{-\infty}^{\infty}\left|f^{\prime}(x)+e^{i \alpha}\left\{-i \tau f(x)+f^{\prime}(x)\right\}\right|^{p} d x \leqq \tau^{p} \int_{-\infty}^{\infty}|f(x)|^{p} d x, \quad(p \geqq 1) .
$$

Integrating both the sides with respect to $\alpha$ from 0 to $2 \pi$ we get

$$
\begin{array}{r}
\int_{0}^{2 \pi} d \alpha \int_{-\infty}^{\infty}\left|f^{\prime}(x)+e^{i \alpha}\left\{-i \tau f(x)+f^{\prime}(x)\right\}\right|^{p} d x \leqq 2 \pi \tau^{p} \int_{-\infty}^{\infty}|f(x)|^{p} d x, \\
\quad(p \geqq 1) .
\end{array}
$$

Note that $f^{\prime}(x)$ can be zero only at a countable number of points. Besides, we can clearly invert the order of integration in the left-hand side of (3.19). Therefore

$$
\begin{aligned}
\int_{0}^{2 \pi} d \alpha \int_{-\infty}^{\infty}\left|f^{\prime}(x)+e^{i \alpha}\left\{-i \tau f(x)+f^{\prime}(x)\right\}\right|^{p} d x \\
=\int_{0}^{2 \pi} d \alpha \int_{-\infty}^{\infty}\left|f^{\prime}(x)\right|^{p}\left|1+e^{i \alpha} \frac{-i \tau f(x)+f^{\prime}(x)}{f^{\prime}(x)}\right|^{p} d x \\
=\int_{-\infty}^{\infty}\left|f^{\prime}(x)\right|^{p} d x \int_{0}^{2 \pi}\left|1+e^{i \alpha} \frac{-i \tau f(x)+f^{\prime}(x)}{f^{\prime}(x)}\right|^{p} d \alpha \\
=\int_{-\infty}^{\infty}\left|f^{\prime}(x)\right|^{p} d x \int_{0}^{2 \pi}\left|1+e^{i \alpha} \frac{B(x)}{A(x)}\right|^{p} d \alpha .
\end{aligned}
$$


Since $h_{f}(\pi / 2)=0$ it follows from (3.2) that for all real $x$

$$
|A(x)|=\left|f^{\prime}(x)\right| \leqq\left|-i \tau f(x)+f^{\prime}(x)\right|=|B(x)|,
$$

i.e. $|B(x) / A(x)| \geqq 1$. Thus for a fixed real $x$ and every $p>0$

$$
\int_{0}^{2 \pi}\left|1+e^{i \alpha} \frac{B(x)}{A(x)}\right|^{p} d \alpha=\int_{0}^{2 \pi}|1+| \frac{B(x)}{A(x)}\left|e^{i \alpha}\right|^{p} d \alpha \geqq \int_{0}^{2 \pi}\left|1+e^{i \alpha}\right|^{p} d \alpha .
$$

This follows, for example, by taking $G(\zeta)=1+\zeta$ and $R=|B(x) / A(x)|$ in the well known inequality

$$
\int_{0}^{2 \pi}\left|G\left(R e^{i \alpha}\right)\right|^{p} d \alpha \geqq \int_{0}^{2 \pi}\left|G\left(e^{i \alpha}\right)\right|^{p} d \alpha, \quad(p>0, R \geqq 1)
$$

valid for every entire function $G(\zeta)$.

Thus we finally get

$$
\int_{0}^{2 \pi}\left|1+e^{i \alpha}\right|^{p} d \alpha \int_{-\infty}^{\infty}\left|f^{\prime}(x)\right|^{p} d x \leqq 2 \pi \tau^{p} \int_{-\infty}^{\infty}|f(x)|^{p} d x, \quad(p \geqq 1)
$$

which is the same as (3.15).

REMARK 2. If $f(z)$ is real for real $z$, then

$$
\left|i \tau f(x)+f^{\prime}(x)\right|=\left|-i \tau f(x)+f^{\prime}(x)\right| .
$$

Thus from (3.16) we get the following inequality of Duffin and Schaeffer [7]

$$
\left\{\tau^{2}|f(x)|^{2}+\left|f^{\prime}(x)\right|^{2}\right\}^{1 / 2} \leqq \tau \sup _{-\infty<x<\infty}|f(x)|,
$$

valid for entire functions of exponential type $\tau$ which are real for real $z$. The above method also gives an extension of the above inequality to $L_{p}, 1 \leqq p<\infty$. For this, integrate the two sides of (3.18) with respect to $\alpha$ from 0 to $2 \pi$, invert the order of integration and use (3.21) in the inequality so obtained. We shall get the following:

THEOREM 3. If $f(z)$ is an entire function of exponential type $\tau$ belonging to $L_{p}$, $1 \leqq p<\infty$ on the real axis, and real for real $z$, then

$$
\int_{-\infty}^{\infty}\left[\left\{\tau^{2}|f(x)|^{2}+\left|f^{\prime}(x)\right|^{2}\right\}^{1 / 2}\right]^{p} d x \leqq C_{p}(2 \tau)^{p} \int_{-\infty}^{\infty}|f(x)|^{p} d x
$$

where $C_{p}$ is the same as defined in Theorem 2.

It may be pointed out that $C_{p} 2^{p} \rightarrow 1$ as $p \rightarrow \infty$.

If the entire function $f(z)$ of exponential type $\tau$ is periodic on the real axis with period $2 \pi$ then $f(z)$ has the form

$$
f(z)=\sum_{\nu=-n}^{n} a_{\nu} e^{i v z}, \quad n \leqq \tau,
$$

i.e. it must be a trigonometric polynomial. Note the analogy between the above theorem and the following which is certainly best possible [11]. 
THEOREM B. If $f(z)$ is an entire function of exponential type $\tau$ periodic on the real axis with period $2 \pi$, and real for real $z$, then for $1 \leqq p<\infty$

$$
\int_{-\pi}^{\pi}\left[\left\{\tau^{2}|f(x)|^{2}+\left|f^{\prime}(x)\right|^{2}\right\}^{1 / 2}\right]^{p} d x \leqq C_{p}(2 \tau)^{p} \int_{-\pi}^{\pi}|f(x)|^{p} d x,
$$

with the same definition of $C_{p}$.

REMARK 3. By applying Theorem 2 to the function $\omega(z)=e^{i \tau z} \bar{f}(z)$ we can easily deduce with the help of Minkowski's inequality that if $f(z)$ is an entire function of order 1 type $\tau$ belonging to $L_{p}, 1 \leqq p<\infty$ on the real axis with $h_{f}(-\pi / 2)=\tau$, $h_{f}(\pi / 2) \leqq 0$, and $f(z) \neq 0$ for $y<0$, then

$$
\left\|f^{\prime}\right\|_{p} \geqq\left(1-C_{p}^{1 / p}\right) \tau\|f\|_{p} .
$$

If $f(z) \in L_{p}, 1 \leqq p<\infty$ on the real line then it is bounded there and so $h_{f}(\pi / 2) \leqq 0$ implies that $h_{f}(-\pi / 2)$ is necessarily $\tau$. Hence we can drop the restriction $h_{f}(-\pi / 2)$ $=\tau$. However, this result appears to be far from being precise.

4. Let $p_{n}(z)$ be a polynomial of degree $n$ such that $\left|p_{n}(z)\right| \leqq M$ for $|z|=1$. It is easy to verify that $p_{n}\left(e^{i z}\right)$ is an entire function $f(z)$ of exponential type $n$ such that $h_{f}(-\pi / 2)=n$ and $|f(x)| \leqq 1$ for real $x$. If $p_{n}(z)$ has no zeros in $|z|<1, f(z)$ has no zeros in $y>0$, and moreover (since $\left.p_{n}(0) \neq 0\right) h_{f}(\pi / 2)=0$. The method of $\S 3$ can therefore be specialized to give simpler proofs of the following results ([1], [9]).

Let $p_{n}(z)$ be a polynomial of degree $n$ not having zeros in $|z|<1$. If $\left|p_{n}(z)\right| \leqq M$ for $|z|=1$ then for $|z|=R>1$

$$
\left|p_{n}(z)\right| \leqq M\left(R^{n}+1\right) / 2
$$

for $|z|=1$

$$
\left|p_{n}^{\prime}(z)\right| \leqq M n / 2 \text {. }
$$

The best possible estimate for $\int_{0}^{2 \pi}\left|p_{n}^{\prime}\left(e^{i \theta}\right)\right|^{\delta} d \theta$ in terms of $\int_{0}^{2 \pi}\left|p_{n}\left(e^{i \theta}\right)\right|^{\delta} d \theta$ was found out by De Bruijn [5, Theorem 13] but such a result is not obtainable from Theorem 2. Nevertheless, we shall prove the result of De Bruijn in very much the same way as Theorem 2 .

DE BRUIJN'S THEOREM. If the polynomial $p_{n}(z)$ of degree $n$ has no zeros for $|z|<1$, then for $\delta \geqq 1$

$$
\int_{0}^{2 \pi}\left|p_{n}^{\prime}\left(e^{i \theta}\right)\right|^{\delta} d \theta \leqq C_{\delta} n^{\delta} \int_{0}^{2 \pi}\left|p_{n}\left(e^{i \theta}\right)\right|^{\delta} d \theta
$$

where $C_{\delta}=2 \pi / \int_{0}^{2 \pi}\left|1+e^{i \alpha}\right|^{\delta} d \alpha$.

For our proof we need the following:

Lemma 3. Let $\mathscr{P}_{n}$ denote the linear space of polynomials

$$
p_{n}(z)=a_{0}+\cdots+a_{n} z^{n}
$$


of degree $\leqq n$ with complex coefficients, normed by $\left\|p_{n}\right\|=\max \left|p_{n}\left(e^{i \theta}\right)\right|$. Define the linear functional $L$ on $\mathscr{P}_{n}$ as

$$
L: p_{n} \rightarrow l_{0} a_{0}+\cdots+l_{n} a_{n}
$$

where the $l_{i}$ are complex numbers. If the norm of the functional is $N$ then

$$
\int_{0}^{2 \pi} \Theta\left(\frac{\left|\sum_{k=0}^{n} l_{k} a_{k} e^{i k \theta}\right|}{N}\right) d \theta \leqq \int_{0}^{2 \pi} \Theta\left(\left|\sum_{k=0}^{n} a_{k} e^{i k \theta}\right|\right) d \theta
$$

where $\Theta(t)$ is a nondecreasing convex function of $t$.

This result was proved by $H$. S. Shapiro [13, Theorem 8] for the case $\Theta(t)=t$.

Proof of Lemma 3. According to a theorem of Shapiro [13, Theorem 3] $L$ can be represented in the form $L\left[p_{n}(z)\right]=\sum_{k=1}^{m} u_{k} p_{n}\left(z_{k}\right)$, for all $p_{n} \in \mathscr{P}_{n}$ where $z_{1}, \ldots, z_{m}$ are distinct numbers of moduli 1 and $\sum_{k=1}^{m}\left|u_{k}\right|=N$. Let $\zeta$ be any number of modulus one, and apply this to the polynomial $p_{n}(\zeta z)$. We get

$$
\left|\sum_{k=0}^{n} l_{k} a_{k} \zeta^{k}\right|=\left|\sum_{k=1}^{m} u_{k} p_{n}\left(z_{k} \zeta\right)\right| \leqq \sum_{k=1}^{m}\left|u_{k}\right|\left|p_{n}\left(z_{k} \zeta\right)\right| .
$$

Hence if $\Theta(t)$ is a nondecreasing convex function of $t$ then by Jensen's inequality [15, Vol. I, p. 21],

$$
\Theta\left(\frac{\left|\sum_{k=0}^{n} l_{k} a_{k} \zeta^{k}\right|}{N}\right) \leqq \Theta\left\{\frac{\sum_{k=1}^{m}\left|u_{k}\right|\left|p_{n}\left(z_{k} \zeta\right)\right|}{\sum_{k=1}^{m}\left|u_{k}\right|}\right\} \leqq \frac{\sum_{k=1}^{m}\left|u_{k}\right| \Theta\left\{\left|p_{n}\left(z_{k} \zeta\right)\right|\right\}}{\sum_{k=1}^{m}\left|u_{k}\right|}
$$

Setting $\zeta=e^{i \theta}$ and integrating both the sides with respect to $\theta$ from 0 to $2 \pi$ we get the result.

Proof of DeBruijn's theorem. If $\left|p_{n}(z)\right| \leqq M$ for $|z| \leqq 1$ then for $|\lambda|>1$ the polynomial $P(z)=p_{n}(z)-\lambda M$ does not vanish in $|z| \leqq 1$. Let

$$
Q(z)=z^{n} \bar{P}(1 / z)=z^{n} \bar{p}_{n}(1 / z)-z^{n} \bar{\lambda} M=q_{n}(z)-z^{n} \lambda M .
$$

The polynomial $Q(z)$ has all its zeros in $|z|<1$ and $|Q(z)|=|P(z)|$ for $|z|=1$. By another theorem of De Bruijn [5, Theorem 2] it follows that $\left|P^{\prime}(z)\right| \leqq\left|Q^{\prime}(z)\right|$ for $|z|=1$. Thus for $0 \leqq \theta<2 \pi$

$$
\left|d p_{n}\left(e^{i \theta}\right) / d \theta\right| \leqq\left|d q_{n}\left(e^{i \theta}\right) / d \theta-i n e^{i n \theta} \bar{\lambda} M\right| .
$$

By choosing arg $\lambda$ suitably we shall obtain

$$
\left|d p_{n}\left(e^{i \theta}\right) / d \theta\right| \leqq n|\lambda| M-\left|d q_{n}\left(e^{i \theta}\right) / d \theta\right| .
$$

Since the above inequality is true for every $\lambda$ such that $|\lambda|>1$ we get

But clearly

$$
\left|d p_{n}\left(e^{i \theta}\right) / d \theta\right|+\left|d q_{n}\left(e^{i \theta}\right) / d \theta\right| \leqq M n .
$$

$$
\left|d q_{n}\left(e^{i \theta}\right) d \theta\right|=\left|d\left\{e^{-i n \theta} p_{n}\left(e^{i \theta}\right)\right\} / d \theta\right|,
$$

and therefore

$$
\left|d p_{n}\left(e^{i \theta}\right) / d \theta\right|+\left|-i n p_{n}\left(e^{i \theta}\right)+d p_{n}\left(e^{i \theta}\right) / d \theta\right| \leqq M n .
$$


Hence for every $\alpha$ such that $0 \leqq \alpha<2 \pi$, we have

$$
\left|d p_{n}\left(e^{i \theta}\right) / d \theta+e^{i \alpha}\left\{-i n p_{n}\left(e^{i \theta}\right)+d p_{n}\left(e^{i \theta}\right) / d \theta\right\}\right| \leqq M n,
$$

or

$$
\left|\left(e^{i \alpha}+1\right) d p_{n}\left(e^{i \theta}\right) / d \theta-i n e^{i \alpha} p_{n}\left(e^{i \theta}\right)\right| \leqq M n .
$$

Thus the norm of the bounded linear functional

$$
L: p_{n} \rightarrow\left[\left(e^{i \alpha}+1\right) d p_{n}\left(e^{i \theta}\right) / d \theta-i n e^{i \alpha} p_{n}\left(e^{i \theta}\right)\right]_{\theta=0}
$$

is $n$ and it follows from Lemma 3 that for every $\delta \geqq 1$,

$$
\int_{0}^{2 \pi}\left|\frac{d}{d \theta} p_{n}\left(e^{i \theta}\right)+e^{i \alpha}\left\{-i n p_{n}\left(e^{i \theta}\right)+\frac{d}{d \theta} p_{n}\left(e^{i \theta}\right)\right\}\right|^{\delta} d \theta \leqq n^{\delta} \int_{0}^{2 \pi}\left|p_{n}\left(e^{i \theta}\right)\right|^{\delta} d \theta .
$$

The polynomial $p_{n}(z)$ of De Bruijn's theorem has no zeros in $|z|<1$ and therefore [5, Theorem 2]

$$
\left|d p_{n}\left(e^{i \theta}\right) / d \theta\right| \leqq\left|d q_{n}\left(e^{i \theta}\right) / d \theta\right|=\left|-i n p_{n}\left(e^{i \theta}\right)+d p_{n}\left(e^{i \theta}\right) / d \theta\right| .
$$

The proof can now be completed in the same way as for Theorem 2.

5. An operator preserving inequalities between polynomials. Consider the operator $B$ which carries

into

$$
p_{n}(z)=\sum_{v=0}^{n} a_{v} z^{v}
$$

$$
B\left[p_{n}(z)\right]=\lambda_{0} p_{n}(z)+\lambda_{1}(n z / 2) p_{n}^{\prime}(z) / 1 !+\lambda_{2}(n z / 2)^{2} p_{n}^{\prime \prime}(z) / 2 !
$$

where $\lambda_{0}, \lambda_{1}$, and $\lambda_{2}$ are such that all the zeros of

$$
u(z)=\lambda_{0}+{ }^{n} C_{1} \lambda_{1} z+{ }^{n} C_{2} \lambda_{2} z^{2}
$$

lie in the half plane $|z| \leqq|z-n / 2|$.

We start with the following observation.

THEOREM 4. If $p_{n}(z)$ is a polynomial of degree $n$ then

implies

$$
\left|p_{n}(z)\right| \leqq M, \quad|z|=1,
$$

$$
\left|B\left[p_{n}(z)\right]\right| \leqq M\left|B\left[z^{n}\right]\right|, \quad|z| \geqq 1 .
$$

For this we need the following lemma [10, p. 65, see Corollary (18.3)].

LEMMA 4. If all the zeros of a polynomial $P(z)$ of degree $n$ lie in the circle $|z| \leqq 1$ then all the zeros of the polynomial $B[P(z)]$ also lie in the circle $|z| \leqq 1$.

Proof of Theorem 4. If $\lambda$ is a complex number such that $|\lambda|>1$ then $\left|p_{n}(z)\right|<$ $\left|\lambda M z^{n}\right|$ for $|z|=1$. Since the function $\lambda M z^{n}$ has all its zeros inside the unit circle, it follows from Rouchés theorem that the function $p_{n}(z)-\lambda M z^{n}$ has all its zeros 
inside the unit circle. Now let us apply Lemma 4 to the polynomial $P(z) \equiv p_{n}(z)$ $-\lambda M z^{n}$. The operator $B$ is linear, therefore

$$
B\left[p_{n}(z)\right]-\lambda M B\left[z^{n}\right] \equiv B\left[p_{n}(z)-\lambda M z^{n}\right]
$$

has all its zeros inside the unit circle, i.e. it does not vanish for $|z| \geqq 1$. Now if (5.1) is false then there exists $z_{0}$ such that $\left|z_{0}\right| \geqq 1$ and $\left|B\left[p_{n}(z)\right]\right|_{z=z_{0}}>M\left|B\left[z^{n}\right]\right|_{z=z_{0}}$. It is clear that $\left(B\left[z^{n}\right]\right)_{z=z_{0}}=u(n / 2) z_{0}^{n} \neq 0$. We can therefore choose $\lambda$ such that $|\lambda|>1$ and $\left(B\left[p_{n}(z)\right]\right)_{z=z_{0}}-\lambda M\left(B\left[z^{n}\right]\right)_{z=z_{0}}=0$. But this contradicts the fact that $B\left[p_{n}(z)\right]-\lambda M B\left[z^{n}\right]$ does not vanish for $|z| \geqq 1,|\lambda|>1$. Hence

$$
\left|B\left[p_{n}(z)\right]\right| \leqq M\left|B\left[z^{n}\right]\right|
$$

for $|z| \geqq 1$ and Theorem 4 is proved.

If $q_{n}(z)=z^{n} \bar{p}_{n}(1 / z)$ then $\left|q_{n}(z)\right|=\left|p_{n}(z)\right|$ for $|z|=1$. Hence if $p_{n}(z) \neq 0$ in $|z|<1$ then $q_{n}(z) / p_{n}(z)$ is analytic on and inside the unit circle and $\left|q_{n}(z) / p_{n}(z)\right|=1$ on the boundary. By the maximum modulus principle $\left|q_{n}(z)\right| \leqq\left|p_{n}(z)\right|$ for $|z| \leqq 1$ or $\left|z^{n} \bar{q}_{n}(1 / z)\right| \leqq\left|z^{n} \bar{p}_{n}(1 / z)\right|$ for $|z| \geqq 1$. Noting that $z^{n} \bar{q}_{n}(1 / z) \equiv p_{n}(z)$ we get $\left|p_{n}(z)\right|$ $\leqq\left|q_{n}(z)\right|$ for $|z| \geqq 1$. From this it follows that for every $\lambda$ such that $|\lambda|>1$ the polynomial $p_{n}(z)-\lambda q_{n}(z)$ has all its zeros in $|z| \leqq 1$. By Lemma 4 the polynomial $B\left[p_{n}(z)\right]-\lambda B\left[q_{n}(z)\right]$ has no zeros in $|z|>1$. This implies that

$$
\left|B\left[p_{n}(z)\right]\right| \leqq\left|B\left[q_{n}(z)\right]\right| \text { for }|z| \geqq 1 \text {. }
$$

If $p_{n}(z)$ is a polynomial of degree $n$ such that $\left|p_{n}(z)\right| \leqq M$ for $|z| \leqq 1$ then for $0 \leqq \alpha<2 \pi$ the polynomial $p_{n}(z)-M e^{i \alpha}$ does not vanish inside the unit circle. Hence from (5.2) applied to the polynomial $p_{n}(z)-M e^{i \alpha}$ we get

$$
\left|B\left[p_{n}(z)\right]-M \lambda_{0} e^{i \alpha}\right| \leqq\left|B\left[q_{n}(z)\right]-M e^{-i \alpha} B\left[z^{n}\right]\right|
$$

for $|z| \geqq 1$. Now choose $\alpha$ such that

$$
\left|B\left[q_{n}(z)\right]-M e^{-i \alpha} B\left[z^{n}\right]\right|=M\left|B\left[z^{n}\right]\right|-\left|B\left[q_{n}(z)\right]\right| .
$$

(From Theorem 4 we know that $M\left|B\left[z^{n}\right]\right|-\left|B\left[q_{n}(z)\right]\right| \geqq 0$.) We get

$$
\left|B\left[p_{n}(z)\right]\right|-\left|\lambda_{0}\right| M \leqq M\left|B\left[z^{n}\right]\right|-\left|B\left[q_{n}(z)\right]\right|, \quad|z| \geqq 1,
$$

or

$$
\left|B\left[p_{n}(z)\right]\right|+\left|B\left[q_{n}(z)\right]\right| \leqq\left|\lambda_{0}\right| M+M\left|B\left[z^{n}\right]\right|, \quad|z| \geqq 1 .
$$

Substituting for $\left|B\left[z^{n}\right]\right|$ we get

$$
\left|B\left[p_{n}(z)\right]\right|+\left|B\left[q_{n}(z)\right]\right| \leqq M\left\{\left|\lambda_{0}\right|+\left|\lambda_{0}+\lambda_{1} n^{2} / 2+\lambda_{2} n^{3}(n-1) / 8\right|\right\}
$$

for $|z| \geqq 1$. 
Suppose that $S(\theta)=\sum_{v=-n}^{n} a_{v} e^{i v \theta}$ is a trigonometric polynomial of degree $n$ such that $|S(\theta)| \leqq 1$ for $0 \leqq \theta<2 \pi$. Then $e^{i n \theta} S(\theta) \equiv p_{(S)}\left(e^{i \theta}\right)$, where $p_{(S)}(z)$ is a polynomial of degree $2 n$. It is clear that $\left|p_{(S)}(z)\right| \leqq 1$ for $|z| \leqq 1$. For $z=e^{i \theta}$

$$
\begin{aligned}
B\left[p_{(S)}(z)\right]= & \lambda_{0} p_{(S)}(z)+\lambda_{1}(2 n z / 2) p_{(S)}^{\prime}(z) / 1 !+\lambda_{2}(2 n z / 2)^{2} p_{(S)}^{\prime \prime}(z) / 2 ! \\
= & \lambda_{0} e^{i n \theta} S(\theta)+\left(\lambda_{1} / 1 !\right)\left(n e^{i \theta}\right) \frac{d}{d \theta} p_{(S)}\left(e^{i \theta}\right) \frac{d \theta}{d z} \\
& +\left(\lambda_{2} / 2 !\right)\left(n e^{i \theta}\right)^{2} \frac{d}{d \theta}\left(\frac{d}{d \theta} p_{(S)}\left(e^{i \theta}\right) \frac{d \theta}{d z}\right) \frac{d \theta}{d z} \\
= & \lambda_{0} e^{i n \theta} S(\theta)-i \lambda_{1} n\left\{i n e^{i n \theta} S(\theta)+e^{i n \theta} S^{\prime}(\theta)\right\} \\
& -\left(\lambda_{2} / 2 !\right) n^{2} i e^{i \theta} \frac{d}{d \theta}\left\{n e^{i(n-1) \theta} S(\theta)-i e^{i(n-1) \theta} S^{\prime}(\theta)\right\} \\
= & \lambda_{0} e^{i n \theta} S(\theta)-i \lambda_{1} n\left\{i n e^{i n \theta} S(\theta)+e^{i n \theta} S^{\prime}(\theta)\right\} \\
& -i\left(\lambda_{2} / 2 !\right) n^{2} e^{i \theta}\left\{i n(n-1) e^{i(n-1) \theta} S(\theta)+n e^{i(n-1) \theta} S^{\prime}(\theta)\right. \\
\quad & \left.+(n-1) e^{i(n-1) \theta} S^{\prime}(\theta)-i e^{i(n-1) \theta} S^{\prime \prime}(\theta)\right\} \\
= & e^{i n \theta}\left\{\left(\lambda_{0}+\lambda_{1} n^{2}+\lambda_{2} \frac{n^{3}(n-1)}{2}\right) S(\theta)-i\left(\lambda_{1} n+\lambda_{2} \frac{n^{2}(2 n-1)}{2 !}\right) S^{\prime}(\theta)\right. \\
& \left.\quad \lambda_{2} \frac{n^{2}}{2 !} S^{\prime \prime}(\theta)\right\} .
\end{aligned}
$$

Thus if $S(\theta)$ is a trigonometric polynomial of degree $n$ such that $|S(\theta)| \leqq 1$ for $0 \leqq \theta \leqq 2 \pi$ then from Theorem 4

$$
\begin{aligned}
\mid\left\{\lambda_{0}+\lambda_{1} n^{2}+\lambda_{2} \frac{n^{3}(n-1)}{2}\right\} S(\theta)-i\left\{\lambda_{1} n+\lambda_{2} \frac{n^{2}(2 n-1)}{2}\right\} & S^{\prime}(\theta)-\lambda_{2} \frac{n^{2}}{2} S^{\prime \prime}(\theta) \mid \\
& \leqq\left|\lambda_{0}+2 \lambda_{1} n^{2}+\lambda_{2} n^{3}(2 n-1)\right|
\end{aligned}
$$

if the zeros of the polynomial $\lambda_{0}+{ }^{2 n} C_{1} \lambda_{1} z+{ }^{2 n} C_{2} \lambda_{2} z^{2}$ lie in the half plane $|z| \leqq|z-n|$.

Let us put

$$
\begin{aligned}
-\lambda_{2} n^{2} / 2 & =a, \\
-i\left\{\lambda_{1} n+\lambda_{2} n^{2}(2 n-1) / 2\right\} & =b, \\
\lambda_{0}+\lambda_{1} n^{2}+\lambda_{2} n^{3}(n-1) / 2 & =c,
\end{aligned}
$$

and solve for $\lambda_{0}, \lambda_{1}$, and $\lambda_{2}$. We get $\lambda_{0}=-a n^{2}-i b n+c, \lambda_{1}=\{i b+a(2 n-1)\} / n$ and $\lambda_{2}=-2 a / n^{2}$, so that

$$
\lambda_{0}+2 \lambda_{1} n^{2}+\lambda_{2} n^{3}(2 n-1) \equiv-a n^{2}+i b n+c .
$$

Hence we conclude the following: 
THEOREM 5. If $S(\theta)$ is a trigonometric polynomial of degree $n$ such that $|S(\theta)| \leqq 1$ for $0 \leqq \theta<2 \pi$ and the zeros of the polynomial

$$
u_{1}(z)=2 a(2 n-1) z^{2} / n-2\{a(2 n-1)+i b\} z+a n^{2}+i n b-c
$$

lie in the half plane

$$
|z| \leqq|z-n|
$$

then

$$
\left|a S^{\prime \prime}(\theta)+b S^{\prime}(\theta)+c S(\theta)\right| \leqq\left|-a n^{2}+i b n+c\right| .
$$

If $f(z)=a z^{2}+b z+c$ then (5.5) can be written as

$$
\left|f\left(\frac{d}{d \theta}\right) S(\theta)\right| \leqq \mid f(\text { in }) \mid \text {. }
$$

The zeros of the polynomial $u_{1}(z)$ are given by

$$
z=\frac{n}{2}+\frac{n}{4 a(2 n-1)}\left\{2 i b \pm \frac{2 i}{\sqrt{ } n}\left(n b^{2}+n(2 n-1) a^{2}-2 a c(2 n-1)\right)^{1 / 2}\right\} .
$$

If the constants $a, b$, and $c$ are real and

$$
n b^{2}+n(2 n-1) a^{2}-2 a c(2 n-1) \geqq 0,
$$

then the zeros have the form $z=n / 2 \pm i \beta$ where $\beta$ is purely real, i.e. they lie in the half plane defined by (5.4).

It can be easily verified that if $a z^{2}+b z+c$ is a real quadratic polynomial (linear if $a=0$ ) whose zeros lie in the region $H$ bounded by the two branches of the hyperbola

$$
y^{2}-x^{2} /(2 n-1)=n / 2,
$$

then the coefficients $a, b$, and $c$ satisfy (5.7). Thus if $f(z)$ is a real polynomial whose zeros lie in the region $H$ then it can be expressed as the product of linear and quadratic factors with real coefficients satisfying (5.7). Hence as a particular case of Theorem 5 we get the following:

COROLlaRY 2 [6]. If $f(z)$ is a real polynomial whose zeros lie in the region $H$ and $S(\theta)$ is a trigonometric polynomial of degree $n$ with arbitrary complex coefficients, then for every real $\theta$ we have the inequality

$$
\left|f\left(\frac{d}{d \theta}\right) S(\theta)\right| \leqq \max _{0 \leqq \theta<2 \pi}|S(\theta)| \mid f(\text { in }) \mid .
$$

Taking $\lambda_{0}=\lambda n^{2}, \lambda_{1}=-2 / n, \lambda_{2}=-8 / n^{2}$ in Theorem 4 we get:

COROLlaRY 3. If $p_{n}(z)$ is a polynomial of degree $n>1$ and $\left|p_{n}(z)\right| \leqq 1$ for $|z| \leqq 1$ then

$$
\left|\lambda n^{2} p_{n}(z)-z p_{n}^{\prime}(z)-z^{2} p_{n}^{\prime \prime}(z)\right| \leqq n^{2}|1-\lambda|
$$

if $|\lambda| \leqq \frac{1}{4}(1+1 / n)$.

For if $|\lambda| \leqq \frac{1}{4}(1+1 / n)$ then the zeros of the polynomial

$$
\lambda n^{2}-2 z-4(n-1) z^{2} / n
$$

lie in the half plane defined by $|z| \leqq|z-n / 2|$. 
Since in Corollary $3 \lambda$ is allowed to be complex it is a partial generalization of the following result of Boas [4, see Corollary 2].

If $p_{n}(z)$ is a polynomial of degree $n>1$ and $\left|p_{n}(z)\right| \leqq 1$ for $|z|<1$, then

$$
\left|\lambda n^{2} p_{n}(z)-z p_{n}^{\prime}(z)-z^{2} p_{n}^{\prime \prime}(z)\right|
$$

does not exceed $(1-\lambda) n^{2}$ if $\lambda \leqq 1 / 3+1 /\left(6 n^{2}\right)$, and does not exceed the maximum of $\left|\lambda n^{2} \cos n \theta-\frac{1}{2} n \cot (\theta / 2) \sin n \theta\right|, 0<\theta<\pi$, if $\lambda>1 / 3+1 /\left(6 n^{2}\right)$.

If, in particular, $p(z) \neq 0$ for $|z|<1$ or $p(z)$ is a symmetric polynomial in the sense that $|p(z)|=|q(z)|$ then with the help of (5.2) it follows from (5.3) that the expression on the right-hand side of (5.9) can be replaced by

$$
\frac{1}{2}\left\{n^{2}(|\lambda|+|1-\lambda|)\right\}
$$

which is equal to $n^{2} / 2$ if $0 \leqq \lambda \leqq \frac{1}{4}(1+1 / n)$. Let $S(\theta)$ be a real trigonometric polynomial of degree $n$ and let us apply our conclusion to the symmetric polynomial $P_{(S)}\left(e^{i \theta}\right) \equiv e^{i n \theta} S(\theta)$ of degree $2 n$. We get

$$
\left|4 \lambda n^{2} P_{(S)}(z)-z P_{(S)}^{\prime}(z)-z^{2} P_{(S)}^{\prime \prime}(z)\right| \leqq 2 n^{2} \max _{0 \leqq \theta<2 \pi}|S(\theta)|, \quad 0 \leqq \lambda \leqq \frac{1}{4}(1+1 / n) .
$$

Thus for $0 \leqq \lambda \leqq \frac{1}{4}(1+1 / n)$

i.e.

$$
\left|(4 \lambda-1) n^{2} S(\theta)+2 i n S^{\prime}(\theta)+S^{\prime \prime}(\theta)\right| \leqq 2 n^{2} \max _{0 \leqq \theta<2 \pi}|S(\theta)|,
$$

$$
\left\{(4 \lambda-1) n^{2} S(\theta)+S^{\prime \prime}(\theta)\right\}^{2}+4 n^{2}\left\{S^{\prime}(\theta)\right\}^{2} \leqq 4 n^{4}\left\{\max _{0 \leqq \theta<2 \pi}|S(\theta)|\right\}^{2}
$$

By choosing $\lambda=\frac{1}{4}(1+1 / n)$ or $\lambda=\frac{1}{4}(1-1 / n)$ as the need may be, we can make

$$
\left\{(4 \lambda-1) n^{2} S(\theta)+S^{\prime \prime}(\theta)\right\}^{2}=\left(n|S(\theta)|+\left|S^{\prime \prime}(\theta)\right|\right)^{2}
$$

and then conclude that for a real trigonometric polynomial $S(\theta)$ of degree $n$,

$$
\left\{n|S(\theta)|+\left|S^{\prime \prime}(\theta)\right|\right\}^{2}+4 n^{2}\left|S^{\prime}(\theta)\right|^{2} \leqq 4 n^{4}\left\{\max _{0 \leqq \theta<2 \pi}|S(\theta)|\right\}^{2}
$$

\section{REFERENCES}

1. N. C. Ankeny and T. J. Rivlin, On a theorem of S. Bernstein, Pacific J. Math. 5 (1955), 849-852.

2. R. P. Boas, Jr., Entire functions, Academic Press, New York, 1954.

3. - Inequalities for asymmetric entire functions, Illinois J. Math. 1 (1957), 94-97.

4. - A variational method for trigonometric polynomials, Illinois J. Math. 3 (1959), 1-10.

5. N. G. De Bruijn, Inequalities concerning polynomials in the complex domain. Nederl. Akad. Wetensch. Proc. 50 (1947), 1265-1272.

6. K. Dočev, On a theorem of S. N. Bernstein, Dokl. Akad. Nauk SSSR 146 (1962), 17-19= Soviet Math. Dokl. 3 (1962), 1222-1223.

7. R. J. Duffin and A. C. Schaeffer, Some inequalities concerning functions of exponential type, Bull. Amer. Math. Soc. 43 (1937), 554-556.

8. - Some properties of functions of exponential type, Bull. Amer. Math. Soc. 44 (1938), 236-240.

9. P. D. Lax, Proof of a conjecture of P. Erdös on the derivative of a polynomial, Bull. Amer. Math. Soc. 50 (1944), 509-513. 
10. M. Marden, The geometry of the zeros of a polynomial in a complex variable, Math. Surveys, No. 3, Amer. Math. Soc., Providence, R. I., 1949.

11. Q. I. Rahman, An inequality for trigonometric polynomials, Amer. Math. Monthly 70 (1963), 56-57.

12. - On asymmetric entire functions. II, Math. Ann. 167 (1966), 49-52.

13. H. S. Shapiro, On a class of extremal problems for polynomials in the unit circle, Portugal. Math. 20 (1961), 67-93.

14. E. M. Stein, Functions of exponential type, Ann. of Math. 65 (1957), 582-592.

15. A. Zygmund, Trigonometric series, Cambridge Univ. Press, New York, 1959.

\section{UNIVERSITÉ DE MONTREAL,}

Montréal, Canada 